\title{
Comparative study of prediction methods of gel point of mixed crude oil
}

\author{
Chaofei $\mathrm{Nie}^{1}$, Huanhuan Wang ${ }^{2}$, Hao Lan ${ }^{1}$, Bo Xu ${ }^{1}$, Feng Yan $^{1}$,Qifu Li ${ }^{1}$, Yu Diao ${ }^{1}$ \\ ${ }^{1 .}$ PetroChina Pipeline Research and Development Center, Lang fang, China \\ 2. Southwest Oil an Gas Field Company Northwest of Sichuan Gas Production District, Mianyang, China
}

\begin{abstract}
Gel point is an important parameter of the safe pipeline transportation of the waxy crude oil, and predicting the gel point of the mixed crude oil quickly and precisely has a certain practical significance for this kind of mixed transportation. By adopting the scientific evaluation method of the prediction model, the adaptation of the Model Li Chuangwen, Model Liu Tianyou I, Model Liu Tianyou II, and Model Chen Jun to predicting the gel point of mixed crude oil transported in a certain pipeline is analyzed and compared, by which the most appropriate model for the prediction of the mixed crude oil is confirmed. It is found that Model Liu Tianyou I is most suitable for the mixed crude oil. Therefore, from the engineering point of view, for the mixed crude oil mentioned in this text, the gel point calculated by Model Liu Tianyou I has a certain reference value.
\end{abstract}

Keywords:mixed crude oil; gel point; prediction model; evaluation method.

\section{INTRODUCTION}

The gel point is the highest temperature of crude oil losing fluidity under the test conditions, and it is an important parameter to guide the safe pipeline transportation of waxy crude oil ${ }^{[1]}$. When two components crude oil of different gel points are mixed, the gel point of mixed crude oil is usually between that of two components crude oil, and does not take a simple linear relation with the mix ratio of the two components ${ }^{[2]}$. The ratio of the two components crude oil is subject to changes with practical crude oil production and transportation. While the gel point can be measured by experiment, the testing process is relatively complicated. Therefore, a fast and accurate calculation method of the gel point of mixed crude oil has a certain practical significance for the pipeline process calculation.

\section{COMMON GEL POINT PREDICTION MODEL}

The existing calculation model of mixed crude oil gel point is the empirical or semi-empirical formula derived mainly by the users from inductive experimental data. The existing calculation model is often of good calculation precision for the oil sample used in modeling, but not for the other crude oil. When these models are used, it is necessary to evaluate the applicability of the models first, and select the model of higher accuracy specific to a certain research object.

The common gel point calculation models are Model Li Chuangwen ${ }^{[3-4]}$, Model Liu Tianyou I, Model Liu Tianyou II ${ }^{[5-6]}$ and Model Chen Jun ${ }^{[7]}$. Model Li Chuangwen and Model Liu Tianyou are calculation formula gained by amending the linear model through the experimental data of crude oil and mixed crude oil in the representative single well of the various Xinjiang oil fields. When these two models are used, not only the gel point of each component crude oil but also of each twocomponent crude oil mixed in the same ratio need to be known. In the analysis of the data of gel point of mixed crude oil, Chen Jun found that the absolute value of the difference between the measured value of and the linear weighted method calculated value of the gel point of the two-component mixed crude oil correlates properly with the absolute value of the difference between the gel points of each component crude oil. Therefore, a new method of calculating mixed crude oil gel point is put forward where the gel point of components crude oil mixed in various ratios can be predicted only when the gel point of each component crude oil is known. In this paper, a model suitable for predicting the gel point of the mixed crude oil in a pipeline is selected from the above four models.

The calculation formula of each model is shown in Table I. 
Table I Calculation Model of Gel point of Mixed Crude Oil

\begin{tabular}{|c|c|}
\hline Model & Calculation Formula \\
\hline Li & $T_{G}=\sum_{i=1}^{n}\left(X_{i} T_{i}\right)+\sum_{j=1}^{n-1} \sum_{k=j+1}^{n}\left(B_{j k} C_{j k} X_{j} X_{k}\right)$ \\
& $B_{j k}=\left[\log \left(100 X_{j}\right) / \log \left(100 X_{k}\right)\right]^{\operatorname{sgn}\left(\mathrm{C}_{j k}\right)}$ \\
& $C_{j k}=2\left(2 T_{j k}-T_{j}-T_{k}\right)$ \\
\hline Liu I & $T_{G}=\sum_{i=1}^{n}\left(X_{i} T_{i}\right)+\sum_{j=1}^{n-1} \sum_{k=j+1}^{n}\left(B_{j k} C_{j k} X_{j} X_{k}\right)$ \\
& $B_{j k}=1-\frac{X_{k}}{2}+\frac{X_{j}}{2}$ \\
\hline Liu II & $C_{j k}=2\left(2 T_{j k}-T_{j}-T_{k}\right)$ \\
\hline & $T_{G}=\sum_{i=1}^{n}\left(X_{i} T_{i}\right)+n^{n} X_{1} X_{2} \cdots X_{n}\left(T_{1 / n}-\frac{1}{n} \sum_{j=1}^{n} T_{j}\right)$ \\
& $T_{G}=\sum_{i=1}^{n}\left(X_{i} T_{i}\right)+\sum_{j=1}^{n-1} \sum_{k=j+1}^{n}\left(B_{j k} C_{j k} X_{j} X_{k}\right)$ \\
Chen & $B_{j k}=1-\frac{X_{k}}{2}+\frac{X_{j}}{2}$ \\
& $C_{j k}= \pm 0.698\left|T_{j}-T_{k}\right|^{1.1456}$
\end{tabular}

Note: In the table, $\mathrm{T}_{\mathrm{G}}$ stands for the gel point of mixed crude oil, $\mathrm{X}_{\mathrm{i}}$ stands for the mix proportion of component oil $\mathrm{i}$, $\mathrm{T}_{\mathrm{i}}$ the gel point of the component oil $\mathrm{i}, \mathrm{T}_{\mathrm{jk}}$ the gel point of components oil $\mathrm{j}$ and $\mathrm{k}$ mixed in the same ratio, $\mathrm{n}$ the number of component oil type, $T_{1 / n}$ the gel point of $n$ kinds of components oil mixed in the same ratio.

As can be seen from the table, all the methods above have adopted a hybrid rule method. The main difference is that $\mathrm{Bij}$, modified parameter of the ratio and $\mathrm{Cij}$, modified parameter of mixed oil properties have different calculation methods. If Model Li Chuangwen, Model Liu Tianyou I and Liu Model Tianyou II are used, not only the gel point and the ratio of each component oil but also the gel point of any two components oil mixed in the same ratio need to be known. Nevertheless, under the condition of Model Chen Jun, the gel point of the mixed crude oil can be calculated only when the gel point and the ratio of each component oil are known.

\section{Prediction Model Evaluation Method}

\section{A. Evaluation index}

For the screening of gel point models of mixed crude oil, the following evaluation indexes were selected.

$$
\begin{gathered}
A A D=\frac{1}{n} \sum_{i=1}^{n}\left|G P D_{i}\right| \\
G P D=T_{p}-T_{m}
\end{gathered}
$$

In the formula above, $T_{p}$ is the measured value of gel point and $T_{m}$ is the calculated value of gel point.

GPD is the absolute deviation of the measured value and the calculated value of gel point. ADD, Absolute Average Deviation, is the average value of the absolute deviations calculated in $\mathrm{n}$ kinds of gauging points with the given model.

(2) Maximum Absolute Deviation (MAD)

$$
M A D=\max \left|G P D_{i}\right|
$$

MAD, Maximum Absolute Deviation, is maximum value of the absolute deviations calculated in $n$ kinds of gauging points with the given model.

(3) Absolute Deviation Distribution ( $\mathrm{Da}$ )

$$
D a=\frac{n_{a}}{n}
$$

Da, Absolute deviation distribution, is the ratio of the number of measuring points whose deviation exceeds a certain limit to the total number of measuring points. It is stipulated in SY / T0541-2009 "Measurement Method of Gel point of Crude Oil" that if the same operator using the same equipment in the same lab measures the same oil sample twice under the same experimental conditions, the difference between the two measuring results should not exceed $2{ }^{\circ} \mathrm{C}$. Therefore, na takes the measuring points where the calculation deviation of the model exceeds $2{ }^{\circ} \mathrm{C}$.

The above three evaluation indexes are inverse indexes, that is, the smaller the index value, the better the model.

\section{B. Data Standardized Process}

For evaluation process of the multi-index evaluation, the measurement units and the magnitude of the indexes are not the same, and the significance degree of the comprehensive evaluation of the accuracy of the models is not the same for different indexes, so the statistical index values cannot simply be added. In order to make each index value comparable, the data should be processed first, and then the appropriate method is selected to integrate data.

Data standardization is also the indexation of statistical data. The indexes of different properties directly added cannot correctly reflect the comprehensive results of different forces. Therefore changing the property of the inverse index data has to be considered first so that all the indexes have the same chemotaxis on the evaluation scheme, and then the correct result can be gained by adding the indexes. The data dimensionless processing is mainly to solve the problem of data comparability. After the chemotaxis and dimensionless processing of the data, the original data are converted into the measured values of the dimensionless indexes, that is, the index values are in the same level of magnitude, where a comprehensive evaluation analysis can be conducted. 
There are many methods of data standardization. For the same chemotaxis treatment of inverse index, the threshold value method is used to deal with the data matrix formed by each model index to be evaluated to change the index property. That is,

$$
X=\left(x_{i j}\right)_{m \times \mathrm{n}} \stackrel{\text { 阈值法 }}{\longrightarrow} Y=\left(\mathrm{y}_{\mathrm{ij}}\right)_{\mathrm{m} \times \mathrm{n}}
$$

In the formula:

$\mathrm{X}$ - data matrix made up of the index values;

$\mathrm{Y}$-data matrix of index values after dimensionless treatment;

$\mathrm{m}$ - the number of models;

$\mathrm{n}$ - the number of indexes.

Among them, the conversion rule of the threshold value method matrix is,

$$
\mathrm{y}_{\mathrm{ij}}=\frac{\operatorname{maxx}_{\mathrm{j}=1,2 \cdots \mathrm{m}}-\mathrm{x}_{\mathrm{ij}}}{\operatorname{maxx}_{\mathrm{j}=1,2 \cdots \mathrm{m}}-\min _{\mathrm{j}=1,2 \cdots \mathrm{m}}}(\mathrm{i}=1,2, \cdots \mathrm{k})
$$

In the formula, $\mathrm{i}$ and $\mathrm{j}$ represent the $\mathrm{j}^{\text {th }}$ index of the $\mathrm{i}^{\text {th }}$ model; $\mathrm{m}$ and $\mathrm{k}$ represent the number of indexes and models respectively.

The evaluation value $y_{i j}$ calculated by the formula 6 decreases as $\mathrm{x}_{\mathrm{ij}}$ increases in the interval [0,1]. After the treatment of threshold value method, the three indexes, which are originally the inverse indexes, can be converted into the positive index evaluation values, and the original data can be transformed into the dimensionless index evaluation values, which can be processed by the comprehensive evaluation analysis.

\section{Determining the weight of the indexes}

The significance of each evaluation index varies from model evaluation to model evaluation. To reflect the difference of its function, it is necessary to make the scientific weight adjustment to the valuation indexes to ensure the rationality of the comprehensive evaluation result. The method of determining the weight of indexes mainly includes subjective weighting method and objective weighting method. The subjective weighting method adopts qualitative method, where weights are obtained by experts making subjective judgments according to experience, and then the indexes are evaluated synthetically, such as Analytic Hierarchy Process (AHP), Expert Survey (Delphi), Fuzzy Analysis, Binomial Coefficient and so forth. The advantages of these methods are that experts can determine the sequencing of the indexes more reasonably based on practical problems. The objective weighting method starts with the independent data itself, and the comprehensive evaluation is undertaken based on the correlation between the research indexes of historical data or between the indexes and the evaluation results. These methods include Maximum Entropy Technique, Principal Component Analysis, MultiObjective Planning Method, Standard Deviation method, etc. Although the evaluation results of these methods have a strong mathematical theoretical basis, the method depends heavily on the actual problem domain, versatility and participation of decision-makers are poor, and the calculation methods are more cumbersome.

Considering that the three evaluation indexes selected in this paper do not accord with the characteristics of mutual independence, Expert Survey Method in the subjective weighting method are selected to determine the index weight. It is the Absolute Deviation Distribution ( $\mathrm{Da}$ ) among the three indexes that has the greatest effect on the model. Second only to Absolute Deviation Distribution (Da ), is the Absolute Average Deviation (AAD), and the Maximum Absolute Deviation (MAD) has the smallest effect. The weights of the three indexes are $\omega_{\mathrm{Da}}, \omega_{\mathrm{AAD}}$ and $\omega_{\mathrm{MAD}}$ respectively, then

$$
\begin{gathered}
\omega_{D \mathrm{a}}+\omega_{A A D}+\omega_{M A D}=1 \\
\omega_{D \mathrm{a}}>\omega_{A A D}>\omega_{M A D}
\end{gathered}
$$

It is assumed that the weight of the index value that has the greatest effect on model accuracy is five times that of the least significant one, then

$$
\omega_{D \mathrm{a}}=5 \omega_{M A D}
$$

Combine the formula 7 and 8 to determine the weights of the three indexes were $\omega_{\mathrm{Da}}=0.5, \omega_{\mathrm{AAD}}=0.4, \omega_{\mathrm{MAD}}=0.1$ respectively.

\section{Multi-index comprehensive evaluation method}

The Weighted Average Method is adopted to synthesize the evaluation values of the three indexes of each model. That is, the comprehensive evaluation matrix $\mathrm{E}$ is obtained by multiplying the data matrix $\mathrm{Y}$ after the index transformation and the weight matrix $\omega \mathrm{T}$, seen in formula 10 . The descending sequencing of the comprehensive evaluation values of the matrix is accordance with the relative superiority and inferiority (sequencing) of the model accuracy.

$$
Y \cdot \omega^{T}=E
$$

\section{Prediction Model COMPARison}

In order to verify the calculation accuracy of the above models, gel points of 111 sets of mixed crude oil of different components and mix ratio for are tested, and the experimental results and the calculated values of the gel points of the four models are compared. The comparison results are shown in Fig. 1 to Fig.4, and the statistical results seen in Table II. 


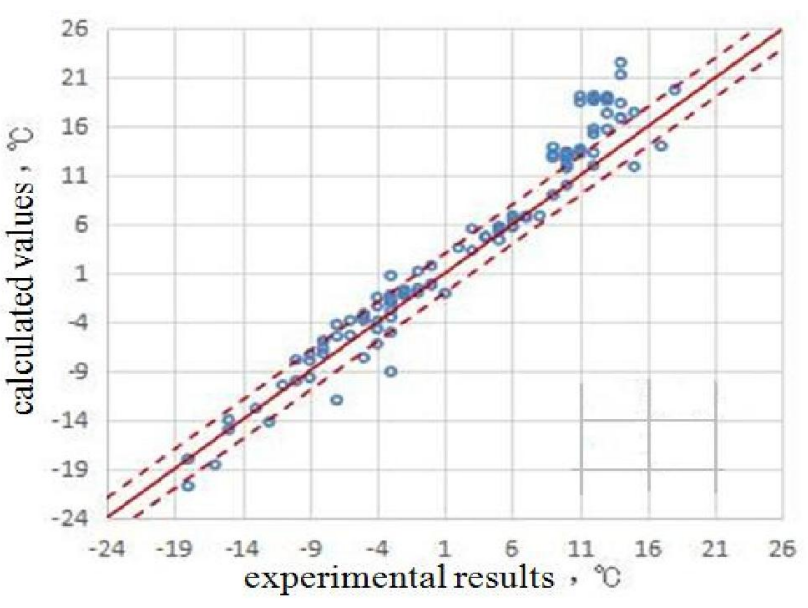

Figure.1 Comparison Between Calculated Value and Measured Value of Model Li Chuangwen

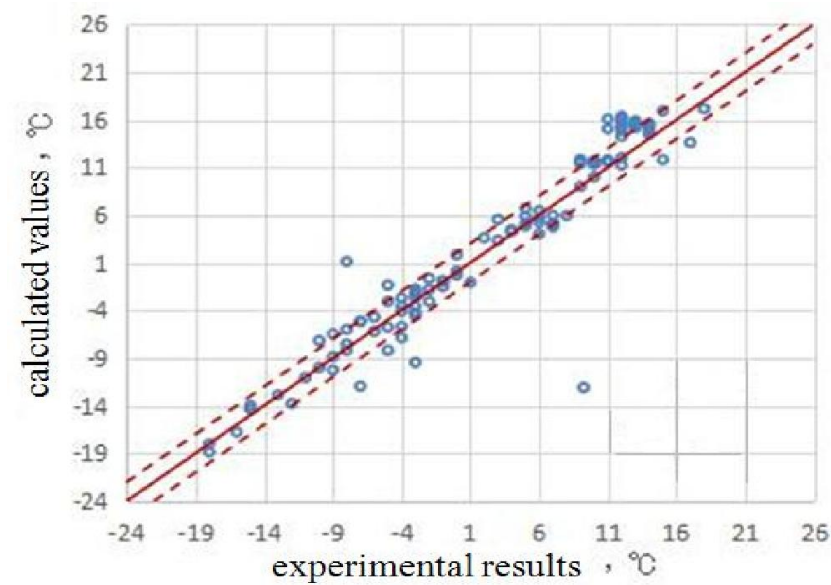

Figure.2 Comparison Between Calculated Value and Measured Value of Model Liu Tianyou I

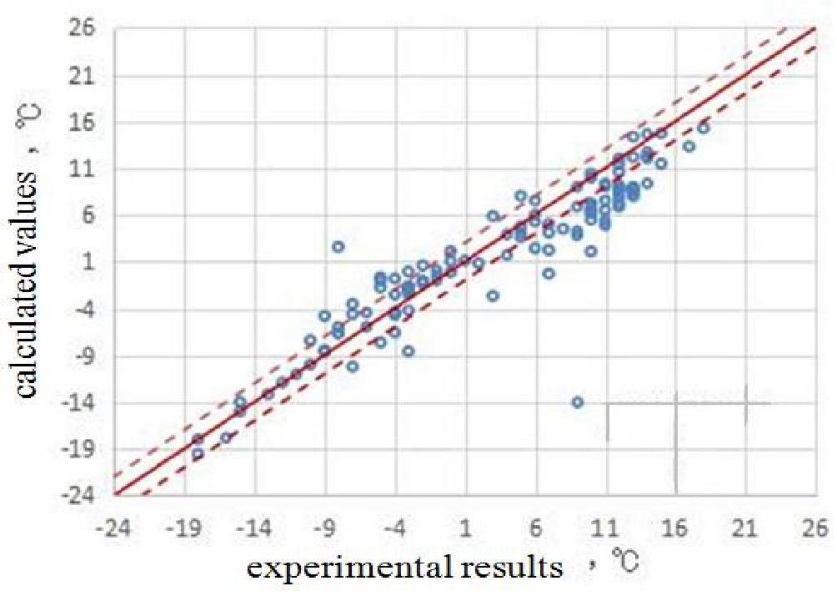

Figure.3 Comparison Between Calculated Value and Measured Value of Model Liu Tianyou II

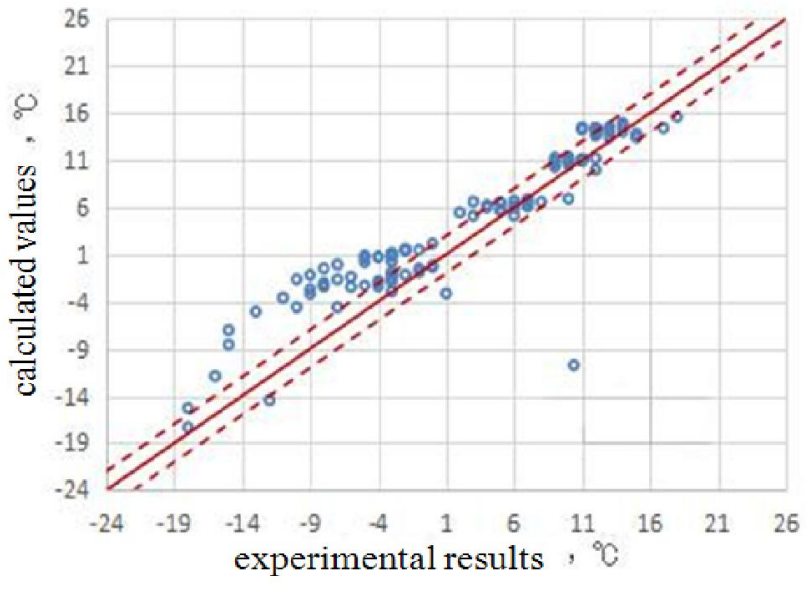

Figure.4 Comparison Between Calculated Value and Measured Value of Model Chen Jun

Table II Statistical Result of Calculation Value of the Four Models

\begin{tabular}{|c|c|c|c|c|}
\hline \multirow{2}{*}{$\begin{array}{c}\text { Evaluation } \\
\text { Index }\end{array}$} & \multicolumn{4}{|c|}{ Statistical Analysis of Calculation Results of the } \\
\cline { 2 - 5 } & Model Li & $\begin{array}{c}\text { Model } \\
\text { Liu I }\end{array}$ & $\begin{array}{c}\text { Model } \\
\text { Liu II }\end{array}$ & $\begin{array}{c}\text { Model } \\
\text { Chen Jun }\end{array}$ \\
\hline AAD & 2.57 & 2.61 & 2.82 & 4.32 \\
\hline MAD & 3.10 & 3.40 & 3.68 & 7.98 \\
\hline Da & 32.35 & 17.65 & 17.65 & 55.88 \\
\hline
\end{tabular}

The contrastive analysis results indicate that there is no significant difference in the Absolute Average Deviation of calculation results between Model Li Chuangwen, and Model Liu Tianyou Modified II and that Model Liu Tianyou Modified I and Model Liu Tianyou Modified II are nearly equivalent in the distribution of both Absolute Average Deviation and Absolute Deviation and that the accuracy of Model Chen Jun is lower than the former three.

According to the above-mentioned model evaluation methods and through scientific calculation, the comprehensive evaluation value of model accuracy is shown in Table III.

Table III Comprehensive Evaluation Results of Gel point Calculation Models of Mixed Crude Oil

\begin{tabular}{|c|c|c|c|c|c|c|c|}
\hline \multirow{2}{*}{ Model } & \multicolumn{3}{|c|}{ X } & \multicolumn{3}{c|}{ Y } & \multirow{2}{*}{ E } \\
\cline { 2 - 7 } & AAD & MAD & Da & AAD & MAD & Da & \\
\hline Liu I & 1.65 & 9.13 & 0.3051 & 1 & 0.66 & 1 & 0.97 \\
\hline Li & 2.40 & 8.55 & 0.4831 & 0.18 & 0.92 & 0 & 0.14 \\
\hline Chen & 2.5 & 8.38 & 0.4746 & 0 & 1 & 0.0478 & 0.13 \\
\hline Liu II & 2.37 & 10.58 & 0.4746 & 0.15 & 0 & 0.0478 & 0.09 \\
\hline
\end{tabular}




\section{CONCLUSIONS}

In summary, based on the comprehensive evaluation results, when the gel point of each component crude oil and of two components crude oil mixed in the same ratio are available, it is recommended to use Model Liu Tianyou Modified I to calculate the gel point of mixed crude oil. Model Li Chuangwen equals Model Chen Jun in the comprehensive evaluation results. Of the four models, Model Liu Tianyou Modified II is least suitable for calculating the gel point of mixed crude oil.

\section{References}

[1] Luo Tanghu. Rheological Properties and Pipeline Transportation Of Waxy Crude Oil [M]. Petroleum Industry Press, 1991.
[2] Yang Xiaoheng, Zhang Guozhong. Design and Management of Oil Pipeline [M]. Petroleum University Press, 1996.

[3] Li Chuangwen, Yan Dafan, Zhang Jinjun. Change of Congealing Points of Crudes and Blends of Xin-jiang Oil Field [J]. Petroleum Planning and Design, 1996 (06):24-27.

[4] Li Chuangwen. A Study of Rheology of Mixed Crude Oil and Its Compatibility Regularity [D]. Beijing: School of Petroleum and Natural Gas Engineering of Petroleum University, 1992.

[5] Liu Tianyou, Zhang Xiujie, Xu Cheng. Calculation of Pour Point of Xinjiang Mixed Oil [J] .Oil and Gas Storage and Transportation, 1993 (2): $37-45$.

[6] Liu Tianyou, Sun Weizhong, Gao Yanqing, Xucheng. Study on the Ordinary Temperature Process of Multi-Blended Crude [J]. Oil and Gas Storage and Transportation, 1999(09):1-6.

[7] Chen Jun, Zhang Jinjun, Zhang Fan. A New Method for Determining Gel Point of Mixed Crude [J]. Journal of the University of Petroleum (Natural Science Edition), 2003 (05):76-80. 